\title{
PD-I/PD-LI blockades in non-small-cell lung cancer therapy
}

This article was published in the following Dove Press journal:

OncoTargets and Therapy

25 January 2016

Number of times this article has been viewed

\author{
Wang Jing ${ }^{1,2, *}$ \\ Miaomiao $\mathrm{Li}^{3, *}$ \\ Yan Zhang ${ }^{2}$ \\ Feifei Teng ${ }^{2}$ \\ Anqin $\mathrm{Han}^{2}$ \\ Li Kong ${ }^{2}$ \\ Hui Zhu² \\ 'Weifang Medical University, Weifang, \\ Shandong Province, People's Republic \\ of China; ${ }^{2}$ Department of Radiation \\ Oncology, Shandong Cancer Hospital \\ and Institute, Jinan, Shandong \\ Province, People's Republic of China; \\ ${ }^{3}$ Shandong Medical College, Jinan, \\ Shandong Province, People's Republic \\ of China
}

*Both these authors contributed equally to the work
Correspondence: Hui Zhu

Department of Radiation Oncology, Shandong Cancer Hospital and Institute, 440 Jiyan Road, Jinan 250I I7, Shandong Province, People's Republic of China

Tel +8653167626112

Fax +86 53। 87984079

Email drzhuhui@।63.com
Abstract: Lung cancer is the leading cause of cancer death in males and the second leading cause of death in females worldwide. Non-small-cell lung cancer (NSCLC) is the main pathological type of lung cancer, and most newly diagnosed NSCLC patients cannot undergo surgery because the disease is already locally advanced or metastatic. Despite chemoradiotherapy and targeted therapy improving clinical outcomes, overall survival remains poor. Immune checkpoint blockade, especially blockade of programmed death-1 (PD-1) receptor and its ligand PD-L1, achieved robust responses and improved survival for patients with locally advanced/metastatic NSCLC in preclinical and clinical studies. However, with regard to PD-1/PD-L1 checkpoint blockade as monotherapy or in combination with other antitumor therapies, such as chemotherapy, radiotherapy (including conventional irradiation and stereotactic body radiotherapy), and target therapy, there are still many unknowns in treating patients with NSCLC. Despite this limited understanding, checkpoint blockade as a novel therapeutic approach may change the treatment paradigm of NSCLC in the future. Here we review the main results from completed and ongoing studies to investigate the feasibility of PD-1/PD-L1 inhibitors, as monotherapy or combinatorial agents in patients with locally advanced and metastatic NSCLC, and explore optimal strategy in such patients.

Keywords: immunotherapy, checkpoint, PD-1, PD-L1, NSCLC

\section{Introduction}

Lung cancer is the leading cause of cancer death in males, and the second leading cause of death in females worldwide. In 2008, a total of 1.6 million cases of lung cancer were diagnosed, and 1.4 million deaths occurred. ${ }^{1}$ Approximately $85 \%$ of lung cancers are non-small-cell lung cancers (NSCLCs), which can be classified in two histological subgroups: squamous cell (Sq) and nonsquamous (non-Sq) cell lung cancer. Traditionally, surgery provides the best chance for cure, but unfortunately, the resection rate is as low as $25 \%-30 \%$. $^{2}$ Emerging evidence suggested that efficacy of stereotactic body radiation therapy (SBRT) for operable NSCLC patients was comparable with surgery; however, the 3-year overall survival (OS) rate was only $76.0 \%$. ${ }^{3}$ Most newly diagnosed NSCLC patients, approximately $85 \%$, cannot undergo surgery because of locally advanced (Stage IIIB; 22\%) or metastatic (Stage IV; 56\%) disease. $^{4-7}$ And according to data from SEER, the corresponding 5-year OS rates were $27.4 \%$ for regional and $4.2 \%$ for distant, even including partial small-cell lung cancer with a poorer prognosis. ${ }^{8}$ Platinum-based chemotherapy, with or without maintenance, was a standard treatment for most patients with advanced NSCLC, while for patients with sensitivity gene mutation, chemotherapy or targeted therapy was the standardized treatment. However, despite the major advances achieved for advanced or metastatic NSCLC over the past decade, survival remains poor, with median survival times of approximately $8-12$ months and the 5 -year survival rate being only $4 \%-5 \%{ }^{5,9,10}$ 
Thus, new therapeutic approaches to improve clinical outcomes are urgently needed.

Immunotherapy, especially immune checkpoint inhibition therapy, is a promising therapeutic approach currently being explored for treatment-naïve or treated patients. The goal of immunotherapy is to recognize and eradicate tumors by restoring the capacity of the host's immune. Effector $\mathrm{T}$-cells played a pivotal role in immunotherapy progression by activation of naïve T-cells; to become fully activated, T-cells rely on two signals: one is the interaction of antigenic peptide-major histocompatibility complex (MHC) with the T-cell receptor (TCR), and the other is provided by the antigen-independent costimulatory signal, which consists of activating signals, such as the engagement of CD28 by B7-1 and B7-2, and inhibitory signals, such as cytotoxic T lymphocyte-associated antigen 4 (CTLA-4) and programmed death-1 (PD-1) receptor pathways. ${ }^{11}$ PD-1 ligand 1 (PD-L1) was highly expressed in many type of cancers or induced by high expression of the cytokine IFN- $\gamma$ secreted by tumor cells (TCs). This engagement of PD-1 and its ligands inhibiting the activation of T-cells may be utilized by TCs to evade immune surveillance. Although PD-L2 blockade can inhibit the function of activated T-cells in theory, an in vivo study showed a greater effect was achieved by targeting the antiPD-L1 blockade compared with anti-PD-L2 blockade. ${ }^{11}$ So far, the targeted therapy on anti-PD-1 and anti-PD-L1 have greatly advanced the treatment of NSCLC.

A series of preclinical and clinical studies indicate that immunotherapy with PD-1/PD-L1 checkpoint blockades achieve robust immune responses and improved clinical outcomes. Checkpoint blockade is considered to be a promising tool for the treatment of lung cancer, combined with chemotherapy or radiotherapy, or combined with targeted therapy. However, there are still many unknowns involving the use of immune checkpoint inhibitors as monotherapy or in combination with other antitumor therapies in patients with NSCLC. Therefore, we reviewed several completed or ongoing trials to discuss the clinical impact of PD-1/PD-L1 blockade on patient outcomes and the potential role for PD-1/PD-L1 inhibitors as a monotherapy or in combination with other therapies.

\section{Mechanism of PD-I and its ligands in cancers and preclinical studies}

The effector cells of tumor immune response mainly include cytotoxic T-lymphocytes, natural killer cells, and macrophages. T-cells are delivered by the binding of the TCR to the antigen peptide-MHC complex, thereby forming an antigen-MHC-TCR complex. T-cells will be completely activated with the help of costimulatory signal, which consists of activating signals, such as the engagement of CD28 by B7-1 and B7-2, and inhibitory signals, such as CTLA-4 and PD-1 pathways. PD-1 is a Type I transmembrane protein and is expressed on T-cells, B-cells, nature killer cells, activated monocytes, and dendritic cells (DCs). PD-L1 and PD-L2, two ligands of PD-1, are also Type I transmembrane proteins and differ in their expression patterns. PD-L1 can be expressed on T-cells, B-cells, DCs, macrophages, a wide variety of nonhematopoietic cells, and also on TCs. ${ }^{11-13}$ PD-1 and its ligands consist of a signal sequence, immunoglobulin (Ig) domain and transmembrane domains, and a short cytoplasmic tail. PD-1 is phosphorylated on its two intracellular tyrosines after PD-1 binding to PD-L1 or PD-L2 based on their IgVlike domain. Then PD-1 binds two phosphatases, SHP-1 and SHP-2, which can bind to the ITIM and ITSM motifs of PD-1, which will downregulate antigen receptor signaling. ${ }^{11,14,15}$ The engagement of PD-1 by PD-L1 and PD-L2 inhibits the function of effector T-cells, while the engagement of B7-1/ B7-2 and its ligand CD28 simulate T-cells proliferation. However, PD-L1, not PD-L2, is a binding partner for B7-1 and competitively binds B7-1 with an affinity greater than CD28, which reduces the function of B7-1-CD28 pathwayactivated T-cells. Furthermore, both PD-L1 and PD-L2 do not bind to B7-2. In addition, PD-L2 expression was restricted to antigen-presenting cells and Th2 cells, and its impact on monitoring T-cells is not as well understood. ${ }^{11,16}$ All of these demonstrate that blocking PD-1 and PD-L1 pathways is more meaningful than blocking PD-L2 pathway.

Numerous preclinical studies showed PD-1/PD-L1 caused dysfunction of tumor-infiltrating lymphocytes (TILs). A preclinical study from Japan showed fewer TILs in PDL1-positive tumor regions compared with PD-L1-negative tumor regions $(P=0.01)$; moreover, the percentage of TILs expressing PD-1 in PD-L1-positive tumor regions was significantly lower than in PD-L1-negative tumor regions $(P=0.02) .{ }^{13}$ Zhang et $\mathrm{al}^{17}$ detected PD-1 expression on $\mathrm{CD}^{+}$ $\mathrm{T}$-cells from normal control peripheral blood mononuclear cells (PBMCs) and the PBMCs of NSCLC patients. Results showed that $\mathrm{CD} 8^{+}$TIL had increased PD-1 expression and impaired immune function. Blockade of PD-1/PD-L1 partially restored the function of T-cells. ${ }^{17}$

\section{Correlation between PD- I/PD-LI expression and clinical activity}

In the era of precision medicine, selection of patients most likely to benefit from specific treatments is vital. Therefore, it 
is particularly important to screen for effective predictive biomarkers. Unfortunately, many questions regarding predictive biomarkers remain unanswered. It is uncertain whether it is the expression of PD-L1 on TCs or immune cells (ICs) that is associated with prognosis. In addition, the correlation of expression level of PD-L1 in TCs or ICs and prognosis is still not clear. Furthermore, the relationship of the efficacy of PD-L1/PD-1 blockade and the pathological types of lung cancer also remains to be determined. Studies regarding PD-L1 expression and associated with overall response rate (ORR) are summarized in Table 1.

Approximately $60 \%$ of NSCLC specimens tested demonstrate PD-L1 expression. ${ }^{18}$ In a recent Phase I study for nivolumab as first-line monotherapy to treat advanced NSCLC patients, the ORR was 31\% in PD-L1-positive tumors, whereas it was $10 \%$ in PD-L1-negative tumors. ${ }^{19}$ Another Phase II study for nivolumab in SqNSCLC patients with greater than or equal to two prior regimens showed $24 \%$ of PD-L1-positive patients (expression more than 5\%) and more than 14\% in PD-L1-negative patients achieved a partial response. ${ }^{20}$ Carbognin et $\mathrm{al}^{21}$ analyzed data from 20 trials, including seven trials regarding NSCLC with 511 patients, and demonstrated higher ORR occurred in PD-L1positive patients $(23.2 \%$ versus $14.5 \%, P=0.0216)$. It seems that response varied with different PD-L1 inhibitors. For nivolumab and pembrolizumab, rather than MPDL3280A, ORR was significantly higher in PD-L1-positive tumors than that in PD-L1-negative tumors.

Another anti-PD-1 drug, pembrolizumab, in a Phase I trial, demonstrated the greatest anticancer efficacy in patients with PD-L1 staining in $\geq 50 \%$ of TCs, with an ORR of $45.2 \% .^{22}$ Updated data showed the ORR was $47 \%$ in the aforementioned group of patients, which was significantly higher than that in patients with PD-L1 staining in $<50 \%$ of TCs. ${ }^{23}$ In a Phase Ia study of MPDL3280A in patients with locally advanced or metastatic solid tumors, including NSCLC, the correlation of ORR and PD-L1 expression on ICs or TCs was analyzed. ${ }^{24}$ PD-L1 positivity was defined as $\geq 5 \%$ of ICs or TCs staining for PD-L1 by immunohistochemistry (IHC). IHC score was ranked 0-3, which was defined as; PD-L1 positive in $<1 \%, \geq 1 \%$ but $<5 \%, \geq 5 \%$ but $<10 \%$, or $\geq 10 \%$ of cells per area, respectively. For patients with NSCLC, PD-L1 positivity determined by IHC was significantly associated with response to MPDL3280A. Updated data presented at the 2015 American Society of Clinical Oncology (ASCO) Annual Meeting showed that the ORR was significantly higher in patients with PD-L1 expression of TC level 3 or IC level $3(45 \%, n=20)$ than that in patients with PD-L1 expression of TC levels $0 / 1 / 2$ and IC levels $0 / 1 / 2(14 \%, n=58) .{ }^{25}$ Another Phase II study (FIR) of MPDL3280A conducted in Stage IIIB/IV NSCLC also indicated that high PD-L1 expression was associated with a higher ORR. ${ }^{26}$ An open-label, nonrandomized, multicenter Phase Ib study is being done to evaluate the safety and efficacy of pembrolizumab (MK-3475) in patients with PD-L1-positive advanced NSCLC. ${ }^{27}$

It seems likely that PD-L1 expression is closely correlated with the curative efficacy of immune checkpoint inhibitors. However, CheckMate 017, a randomized, open-label, international Phase III study of nivolumab

Table I PD-LI expression and association with ORR

\begin{tabular}{|c|c|c|c|c|c|c|c|c|}
\hline \multirow[t]{2}{*}{ Authors } & \multicolumn{2}{|l|}{ ORR (\%) } & \multirow[t]{2}{*}{ Agents } & \multirow[t]{2}{*}{ Pts (no) } & \multirow{2}{*}{$\begin{array}{l}\text { Cutoff value } \\
\text { for PD-LI+ (\%) }\end{array}$} & \multirow{2}{*}{$\begin{array}{l}\text { Detection } \\
\text { methods }\end{array}$} & \multirow[t]{2}{*}{ Antibody } & \multirow{2}{*}{$\begin{array}{l}\text { Evaluation } \\
\text { criterion }\end{array}$} \\
\hline & $\overline{\text { PD-LI }^{+}}$ & PD-LI' & & & & & & \\
\hline Gettinger et al ${ }^{19}$ & 31 & 10 & Nivo & $\begin{array}{l}\text { Treatment-naïve } \\
\text { NSCLC (52) }\end{array}$ & 5 & $\mathrm{IHC}$ & Clone 28-8 & RECIST vI.I \\
\hline Rizvi et al ${ }^{20}$ & 24 (PR) & I4 (PR) & Nivo & $\begin{array}{l}\text { SqNSCLC; } \geq 2 \text { prior } \\
\text { regimens ( } \mid 17)\end{array}$ & 5 & $\mathrm{IHC}$ & ND & Irc-RECIST vI.I \\
\hline Carbognin et $\mathrm{al}^{21}$ & 23.2 & 14.5 & $\begin{array}{l}\text { Nivo } \\
\text { Pembro }\end{array}$ & $\begin{array}{l}\text { First-line or } \geq 2 \text { prior } \\
\text { regimens (5II) }\end{array}$ & $\mathrm{I}$ and 5 & $\mathrm{IHC}$ & ND & RECIST \\
\hline Rizvi et $\mathrm{al}^{23}$ & $\begin{array}{l}47(\geq 50 \%) \\
19(1 \%-49 \%)\end{array}$ & 14 & $\begin{array}{l}\text { MPDL3280A } \\
\text { Pembro }\end{array}$ & $\begin{array}{l}\text { Treatment-naïve } \\
\text { NSCLC (I0I) }\end{array}$ & 1 & $\mathrm{IHC}$ & $22 \mathrm{C} 3$ & RECIST vI.I \\
\hline Horn et $\mathrm{a}^{25}$ & $45(\geq 10 \%)$ & $14(<10 \%)$ & MPDL3280A & $\begin{array}{l}\text { Locally advanced or } \\
\text { metastatic NSCLC (88) }\end{array}$ & 5 & $\mathrm{IHC}$ & SPI42 & RECIST vI.I \\
\hline Spigel et $\mathrm{a}^{26}$ & $\begin{array}{l}29(\geq 10 \%) \\
27(\geq 10 \%) \\
25(\geq 10 \%)\end{array}$ & NR & MPDL3280A & $\begin{array}{l}\text { Treatment-naïv; } \\
\geq 2 \mathrm{~L} \text { without } \mathrm{BM} \\
\geq 2 \mathrm{~L} \text { with treated } \\
\text { asymptomatic } \mathrm{BM}(\mathrm{I} 37)\end{array}$ & 5 & $\mathrm{IHC}$ & SPI42 & RECIST vI.I \\
\hline
\end{tabular}

Abbreviations: ORR, overall response rate; PR, partial response; PD-LI+/-, programmed death ligand I positive/negative; Nivo, nivolumab; Pembro, pembrolizumab; NR, not reported; Pts, patients; no, number of patients; Sq NSCLC, squamous non-small-cell lung cancer; 2L, second-line; BM, brain metastasis; Irc-RECIST, independent radiotherapy review committee-Response Evaluation Criteria in Solid Tumors; IHC, immunohistochemistry; ND, no data. 
versus docetaxel for patients with advanced SqNSCLC during or after failed first-line chemotherapy, indicated that PD-L1 expression was neither prognostic nor predictive of benefit regardless of the expression levels of PD-L1 (1\%, $5 \%$, or $10 \%$ ) as determined by OS and progression-free survival (PFS) rates ${ }^{28}$ Interestingly, however, in previously treated patients with advanced non-SqNSCLC, the study, CheckMate 057, indicated that ORR was significantly higher in the nivolumab arm (31\%) than the docetaxel arm (12\%) at PD-L1 expression level $\geq 1 \% .{ }^{29}$ This phenomenon was also observed between nivolumab and docetaxel arms at PD-L1 levels $\geq 5 \%$ and $\geq 10 \%$. Efficacy of nivolumab may be related to the pathological type of NSCLC, but this is not clear at present. The use of PD-L1 expression status and the expression level of PD-L1 as predictors of the response to checkpoint inhibitors or as biomarkers of prognosis remains controversial. Combined levels of PD-1 and PD-L1 probably predict the prognosis of SqNSCLC. ${ }^{30}$ However, a meta-analysis to investigate correlation between PD-L1 expression and clinicopathological features of NSCLC showed that PD-L1 expression was not associated with histological type, except tumor differentiation. ${ }^{31}$ In another meta-analysis, the correlation between PD-L1 expression and the prognosis of patients with NSCLC was investigated. ${ }^{32}$ Pooled hazard ratio (HR) was 1.75 (95\% confidence interval: $1.40-2.20, P<0.001)$, with significant difference between positive and negative PD-L1 expressions. High level of PD-L1 was associated with increased mortality rates for NSCLC patients. However, this meta-analysis did not provide the correlation between the levels of PD-L1 expression and prognosis.

In addition, there appear to be many factors that may result in inconsistent results. The PD-L1 expression score is defined by IHC methods, which are influenced by subjective factors. In addition, different cutoff values, which were often defined as $1 \%, 5 \%$, and $10 \%$, may lead to inconsistent outcomes. In this era of precision medicine, highly selected patients to participate in these studies are urged. Choosing different biomarkers may be necessary to improve outcomes for different tumors. DNA mismatch repair (MMR) is a system for recognizing and repairing errors of bases that can arise during DNA replication and recombination, as well as repairing some forms of DNA damage. A recent study demonstrated that several immune checkpoint ligands, including PD-L1 and PD-1, were strongly expressed in the MMR-deficient (dMMR) tumor microenvironment. ${ }^{33}$ A Phase II trial that enrolled 41 patients with treatment-refractory progressive metastatic cancer, including eleven patients with dMMR colorectal cancer (CRC), 21 patients with MMR-proficient (pMMR) cancer, and nine patients with dMMR non-CRC, was designed to evaluate the clinical activity of pembrolizumab with a dose of $10 \mathrm{mg} / \mathrm{kg}$ of body weight every 14 days intravenously. ${ }^{34}$ Results showed that the immune-related ORR was $40 \%$ for dMMR CRCs and $0 \%$ for pMMR CRCs; furthermore, the median PFS and OS were not reached in the cohort with dMMR CRC but 2.2 and 5.0 months, respectively, in the cohort with pMMR CRC (HR for death 0.22 , $P=0.05)$. Some studies revealed that $M M R$ gene polymorphisms were associated with lung cancer development. ${ }^{35,36}$ Those studies suggested that MMR may be an effective predictive biomarker of checkpoint inhibitors and a prognostic factor for clinical outcomes.

Response Evaluation Criteria in Solid Tumors (RECIST) was commonly used to evaluate response rate in many trials and was designed to assess solid tumors treated with chemoradiotherapy and targeted treatment. However, most studies have demonstrated that immune response is durable and maintained for a long period of time. RECIST may not accurately describe responses observed because of pseudoprogression. ${ }^{37}$ New criteria have been developed to assess responses to immunotherapy.$^{38}$ Different diagnostic antibodies to assess PD-L1 or PD-1 expression may also impact the observation of ORR, and in addition, different technical staining platforms and different definitions of "positive" IHC staining can still have a significant influence on the analysis of outcomes. ${ }^{39}$

\section{PD-I/PD-LI inhibitors as monotherapy in NSCLC}

In this section, we summarized some Phase I-III clinical trial data that led to use of checkpoint inhibitors, such as nivolumab, pembrolizumab, MPDL3280A, and MEDI4736, as multi-, third-, second-, and first-line therapy. Lung cancer, the second major tumor type so targeted, is the subject of extensive immunotherapy research efforts after the first important and consequential breakthrough made in melanoma. Although lung cancer has been considered to be a nonimmunogenic tumor, the discovery of checkpoint inhibitors has created interest in using immunotherapy in NSCLC. These inhibitors of immune monitoring showed promising efficacy in multiple lines, second-line, and even first-line treatment. Checkpoint blockade research has generated a great deal of interest in the field of immunotherapy. Many monoclonal antibodies (mAbs) have been designed to block this immune checkpoint. Currently, the most commonly used PD-1 inhibitors are nivolumab 
and pembrolizumab, and the major PD-L1 inhibitors are MPDL3280A and MEDI4736 (Table 2). Several clinical research studies have indicated promising efficacy and well-tolerated toxicities. Recently, a meta-analysis was conducted to evaluate the efficacy and safety of anti-PD-1 and anti-PD-L1 mAbs in the treatment of NSCLC. ${ }^{40}$ For patients treated with anti-PD-1 agents, the higher ORR was observed in the median-dose cohort ( $3 \mathrm{mg} / \mathrm{kg}$ ) than that in both low-dose $(1 \mathrm{mg} / \mathrm{kg})$ and high-dose $(10 \mathrm{mg} / \mathrm{kg})$ cohort with significant difference. Rates of common adverse events (AEs) of anti-PD-1 agents were acceptable, including $4.6 \%$ fatigue, $6.7 \%$ gastrointestinal disorders, $11.8 \%$ skin disorders, and 3.2\% pneumonitis. Rates of Grade 3-4 AEs and drug-related death were $16.7 \%$ and $2.5 \%$ for patients with PD-1 inhibition therapy, respectively, whereas it was $31.7 \%$ and $1.8 \%$ for patients with PD-L1 inhibition therapy, respectively. These data indicated that blockade of PD-1/ PD-L1 pathway was safe and effective.

A Phase I trial enrolled 122 advanced NSCLC patients who had received one to five systemic treatment regimens with nivolumab, which was administered at a dose of 1.0 , 3.0 , or $10.0 \mathrm{mg} / \mathrm{kg} .{ }^{41}$ Results showed that the pooled ORR was $18.4 \%$ (14/76), using the modified RECIST. There was no dose-limiting toxicity (DLT), and the response of two patients lasted 1 year or more; for patients with a dose of $3.0 \mathrm{mg} / \mathrm{kg}$, ORR was as high as $32 \%$. Another Phase I, dose-escalation cohort expansion trial enrolled 129 advanced NSCLC patients with one to five prior regimens. ${ }^{42}$ For the entire group, median OS was 9.9 months, whereas it was 14.9 months in the 37 patients receiving $3 \mathrm{mg} / \mathrm{kg}$ nivolumab, 9.5 months in SqNSCLC patients, and 18.2 months in non-SqNSCLC patients. Only $14 \%$ of patients had Grade 3-4 treatment-related AEs, and the most common AE was fatigue ( $3 \%)$, and three patients $(2 \%)$ died of pneumonitis.

As a third-line therapy for locally advanced or metastatic NSCLC, nivolumab has shown promising responses and acceptable toxicity levels. CheckMate 063, a Phase II, singlearm trial, enrolled 117 patients with advanced SqNSCLC who had been treated with greater than or equal to two agents previously. ${ }^{20}$ Nivolumab was administered as an intravenous infusion, $3 \mathrm{mg} / \mathrm{kg}$ every 2 weeks until disease progression or unacceptable AEs occurred. Finally, 17 of 117 patients were assessed for an objective response. At the time of analysis, $13(77 \%)$ patients had an ongoing response and the median duration of response (mDOR) was not reached. Thirty (26\%) of 117 patients had stable disease with a median duration of 6.0 months and the median OS was 8.2 months. Grade 3-4 treatment-related AEs occurred in $17 \%$ of patients; only two patients died of treatment-related pneumonia and ischemic stroke. Early data from CheckMate 153 also showed promising ORR and manageable immune-associated toxicities for those patients who had also been treated with at least two prior systemic regimens. ${ }^{43}$

Nivolumab has also demonstrated significant effectiveness in patients with NSCLC as second-line monotherapy and has been discussed in this section and in the Correlation between PD-1/PD-L1 expression and clinical activity section. To evaluate the safety and efficacy of nivolumab as first-line monotherapy, a Phase I study was conducted; this study enrolled 52 chemotherapy-naïve patients with advanced NSCLC. ${ }^{19}$ At the time the abstract was published, the mDOR was not reached (range: $7.6+, 85.6+$ weeks). The median OS was 98.3 weeks (range: 1.0, 104.4+ weeks); these data were encouraging, irrespective of whether tumor was PD-L1 positive or not. Updated OS and safety data will be presented in the future. As a result of improved outcomes with nivolumab treatment for NSCLC that has progressed while undergoing or after platinum-based chemotherapy, nivolumab became the first PD-1 inhibitor to be approved by the US Food and Drug Administration (FDA) for use in such patients.

Pembrolizumab, which was initially approved for the treatment of melanoma, has received the FDA's breakthrough therapy designation for advanced NSCLC patients without driver genes who have progressed while undergoing or after standard chemotherapy. The data from KEYNOTE-001, which enrolled 495 patients, showed promising outcomes and manageable toxicities. ${ }^{22}$ The pooled ORR was $19.4 \%$ with the use of RECIST version 1.1, with a response rate of $18.0 \%$ and

Table 2 Common PD-I/PD-LI checkpoint inhibitors in clinical use for the treatment of NSCLC

\begin{tabular}{llll}
\hline Drug & Company & Description & Blockade site \\
\hline Nivolumab & Bristol-Myers Squibb & Fully human lgG4 mAb & PD-I on T-cells \\
Pembrolizumab & Merck Sharp \& Dohme Corp & Humanized IgG4 mAb & PD-I on T-cells \\
MPDL3280A & Roche & Human IgGI mAb & PD-LI on TCs \\
MEDI4736 & AstraZeneca & Fully human lgGI mAb & PD-LI on TCs \\
\hline
\end{tabular}

Abbreviations: IgG, Immunoglobulin G; mAb, monoclonal antibody; NSCLC, non-small-cell lung cancer; PD-I, programmed death I; PD-LI, programmed death ligand I; TCs, tumor cells. 
$24.8 \%$ in the previously treated patients and untreated patients, respectively. Median PFS and OS were 3.0 and 9.3 months in treated patients, respectively, compared with 6.0 months and 16.2 months in previously untreated patients. Only $9.5 \%$ of AEs (47/495) less than or equal to Grade 3 were reported and evaluated using the National Cancer Institute Common Terminology Criteria for Adverse Events version 4.0. Now, two randomized, open-label, Phase III studies, KEYNOTE-042 and KEYNOTE-024, were designed to evaluate pembrolizumab as first-line monotherapy compared to platinum-based chemotherapy in PD-L1-positive patients with locally advanced or metastatic NSCLC. ${ }^{44,45}$

MPDL3280A, which was administered intravenously every 3 weeks as monotherapy in patients with incurable advanced NSCLC, also showed a promising ORR of $23 \%(12 / 53) .{ }^{24}$ In POPLAR, a Phase II study comparing MPDL3280A and docetaxel in second-line/third-line NSCLC, reported in the 2015 ASCO meetings, the intentionto-treat interim analysis median OS was 11.4 months for the MPDL3280A group and higher than 9.5 months for the docetaxel group, but the difference was not statistically significant. ${ }^{46}$ Results from subgroup analysis in those patients with PD-L1 expression level of TCs categorized as $1 / 2 / 3$ or ICs categorized as $1 / 2 / 3$ revealed that the interim median OS was not reached for the MPDL3280A group but was 9.1 months for the docetaxel group $(P=0.024)$. Now, another Phase II study, the ATLANTIC, has been designed to evaluate the efficacy of MEDI4736 as a third-line therapy in patients with locally advanced or metastatic NSCLC. ${ }^{47}$ A Phase III study, the OAK, is investigating MPDL3280A in comparison with docetaxel as second-line treatment, and a Phase II study, BIRCH, is recruiting participants to observe the response of MPDL3280A as first-, second-, and third-line therapy for PD-L1-positive patients with locally or metastatic NSCLC. ${ }^{48}$ PD-1/PD-L1 inhibitors used as monotherapy to treat NSCLC showed the therapeutic effectiveness. Several studies that combine PD-1/PD-L1 with chemoradiotherapy and tyrosine kinase inhibitor (TKI)-targeted therapy also showed promising results, which will be discussed in subsequent sections. Completed Phase III and ongoing Phase II/III studies regarding PD-1/PD-L1 checkpoint inhibitors in NSCLC are summarized in Tables 3 and 4, respectively.

\section{PD- I/PD-LI in combination with chemotherapy in NSCLC}

Platinum-based doublet chemotherapy was the standard of care for nononcogene-driven advanced NSCLC, with ORR approximately $30 \%$ and median OS of 8-10 months.

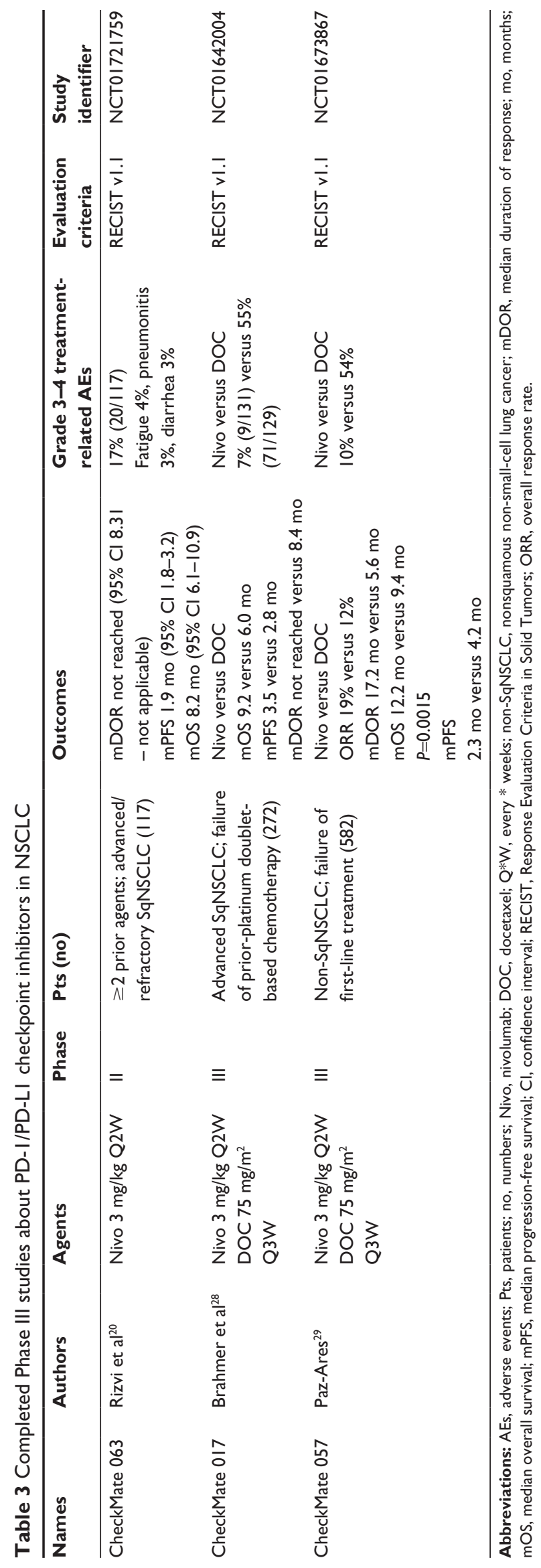




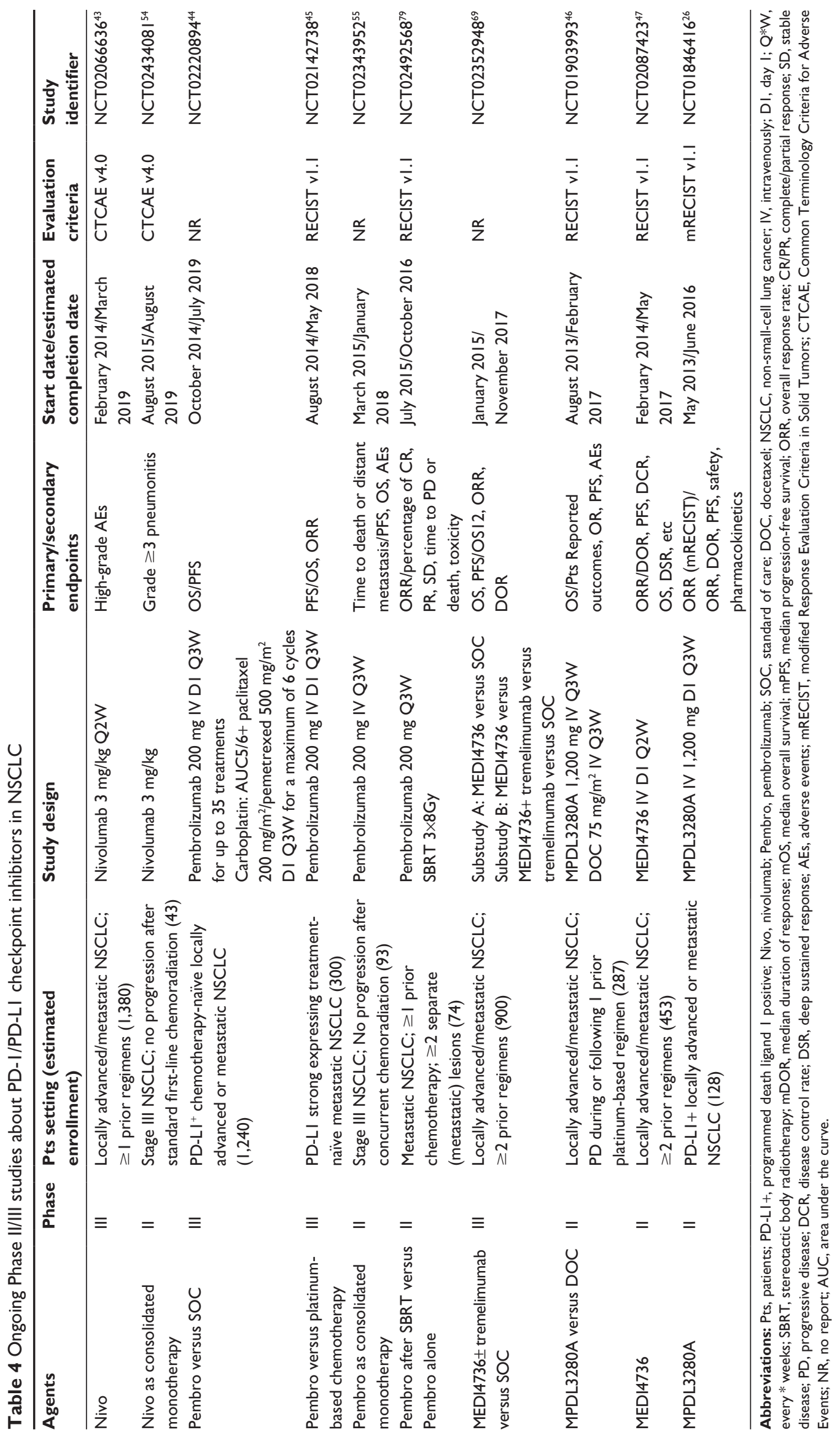


However, treatment options were limited for some patients, such as those with poor performance status. Furthermore, some agent-associated toxicities are so significant that some patients cannot tolerate this therapy. Immunotherapy is a novel therapeutic strategy for the treatment of lung cancer. Immunotherapy responses are slow but durable, whereas chemotherapy can rapidly shrink tumors. In theory, dual therapy that combined chemotherapy with a PD-1/PD-L1 blockade could achieve more long-term control of tumor growth through prolonging PFS, and possibly translate into increased OS. Major progress had been achieved by PD-1/ PD-L1 inhibitors in patients with advanced NSCLC. As a result, checkpoint blockade therapy combined with chemotherapy brings new hope for improved outcomes for advanced lung cancer patients.

Some studies have demonstrated great response and good tolerance on combination therapy. ${ }^{49-51}$ The KEYNOTE 021 study evaluated the safety, tolerability, and clinical activity of pembrolizumab ( 2 or $10 \mathrm{mg} / \mathrm{kg}$ intravenously every 3 weeks) + carboplatin combined with paclitaxel (cohort A) and pemetrexed (cohort C). ${ }^{49}$ The study enrolled 44 patients (20 in cohort A and 24 in cohort C) with treatment-naïve advanced NSCLC. The ORR was 30\% and 58\% in cohorts A and C, respectively. One case of DLT was observed. Rate of Grade 3-4 treatment-associated AEs was 27\%, and no treatment-related deaths occurred. No data on the OS and PFS were reported. However, the data reported still indicated that pembrolizumab combined with chemotherapy as first-line therapy for advanced NSCLC showed an acceptable safety profile and is worth evaluating in a large cohort. MPDL3280A (15 mg/kg intravenously every 3 weeks) in combination with chemotherapy as first-line therapy also showed promising clinical activity in this setting, with well-tolerated toxicities. ${ }^{50}$ ORR was $67 \%$ in the 37 patients evaluated. The major drug-related toxicities included Grade 3-4 AEs of anemia (7\%), neutropenia (7\%-13\%), and thrombocytopenia (7\%), while only one Grade $5 \mathrm{AE}$ of candidemia was observed. Phase III studies are ongoing. Nivolumab, combined with chemotherapy, also showed similar outcomes. The results of the study "CheckMate 012", which were reported at the 2014 ASCO Annual Meeting, were as follows: ORR 33\%-47\%, median PFS 21.0-31.0 weeks, and 1 -year OS rate $50 \%-87 \%$. No treatment-related deaths occurred, but a treatment-related toxicity rate of $45 \%$ at Grade 3/4 was reported. ${ }^{51}$

However, the efficacy of checkpoint inhibitors in combination with chemotherapy for advanced NSCLC was controversial, such as the dose of drugs, timing windows, and so on. Recently, numerous studies designed to evaluate the efficacy of PD-1/PD-L1 inhibitors in combination with chemotherapy for advanced NSCLC patients have been conducted. Some Phase I/II studies are designed to observe pembrolizumab in combination with chemotherapy for patients with advanced NSCLC. ${ }^{52,53}$ A trial, which was designed to evaluate the feasibility of nivolumab consolidation in Stage IIIA/B NSCLC patients with disease progression after standard first-line chemoradiotherapy, is now recruiting participants. ${ }^{54}$ Another study about consolidation therapy with pembrolizumab is recruiting participants with NSCLC who have received chemoradiation. ${ }^{55}$ We hope those studies will generate data that will result in major progress for the treatment of NSCLC.

\section{PD-I/PD-LI in combination with targeted therapy in NSCLC}

Targeted therapies were performed for a subpopulation of NSCLC patients. Epidermal growth factor receptor (EGFR) TKIs, such as gefitinib, erlotinib, and afatinib, were recommended as first-line treatment for patients with EGFRmutant advanced NSCLC. Regardless of prolonged disease control, the 5-year survival rates remain poor for advanced NSCLC. The combination of a PD-1 pathway blockade and EGFR-TKIs might be a promising therapeutic treatment for patients with NSCLC, as data from preclinical studies have implied that EGFR activation upregulated PD-L1, which can contribute to immune evasion. ${ }^{56,57}$ Increasing evidence has demonstrated the correlation of genetic alterations in oncogenes and PD-L1 expression. However, there is still confusion regarding the correlation of PD-L1 status, driver genes (EGFR mutation or ALK rearrangement), and prognosis.

A retrospective study of 125 patients with NSCLC, including EGFR-mutant, KRAS-mutant, ALK-translocated or wild-type EGFR, ALK, and KRAS patients, and with confirmed PD-L1 expression, was correlated with EGFR mutations. ${ }^{58}$ PD-1-positive ( $>5 \%$ of TCs) tumors were significantly associated with the presence of KRAS mutation $(P=0.006)$, whereas PD-L1-positive tumors were significantly associated with the presence of EGFR mutation $(P=0.001)$. Sensitivity to EGFR-TKIs was higher in PD-L1-positive patients than in PD-L1-negative patients in terms of the response rate ( $P=0.01)$. Of note, $\mathrm{PD}-\mathrm{L} 1$-positive tumors were significantly associated with adenocarcinoma histology $(P=0.005)$. Recently, a retrospective study of 56 patients with EGFR-mutant-positive advanced lung adenocarcinoma demonstrated a significantly greater diseasecontrol rate $(P=0.004)$, longer PFS $(P=0.001)$, and OS $(P=0.004)$ after TKIs therapy in PD-L1-positive patients. ${ }^{59}$ Another retrospective study collected data on 170 patients 
with advanced NSCLC treated with EGFR-TKIs in order to examine the correlation of PD-L1, clinicopathologic factors, and prognosis. ${ }^{60}$ Subgroup analyses indicated that PD-L1 expression was associated with significantly shorter OS in EGFR wild-type patients $(P=0.029)$ but not in EGFRmutant patients $(P=0.932)$ treated with EGFR-TKIs for lung adenocarcinoma.

On the other hand, EGFR-TKIs, in combination with PD-L1 blockade were well tolerated in patients with NSCLC. MEDI4736 (cohort A: 3 mg/kg every 2 weeks; cohort B: 10 $\mathrm{mg} / \mathrm{kg}$ every 2 weeks) with gefitinib was utilized in an escalation Phase I study, and the data indicated that the maximum tolerated dose was not reached and no DLTs were observed; Grade 3-4 AEs were observed in three patients, including dyspnea/hypoxia, myalgia/fatigue, and elevated ALT. ${ }^{61}$ These data suggest a need to further evaluate the efficacy of combined therapies in patients with EGFR-sensitized, mutation-positive, TKI-naïve NSCLC.

Antiangiogenesis therapy is one of the most commonly used treatment strategies for advanced lung cancer. Bevacizumab (BEV) is a recombinant $\mathrm{mAb}$ that blocks the vascular endothelial growth factor, and is recommended, in combination with platinum-doublet chemotherapy, for locally advanced or metastatic non-SqNSCLC as first-line therapy or monotherapy maintenance. For patients who have responded (complete response [CR], partial response [PR], or stable disease $[\mathrm{SD}])$ to previous chemotherapy with $\mathrm{BEV}$, maintenance with BEV as monotherapy is recommended. Data from the recent AVAPERL study demonstrated that PFS and median OS for patients treated with single-agent BEV were 3.7 and 13.2 months, respectively. ${ }^{9}$ Recently, a Phase I study evaluated the efficacy of nivolumab $\pm \mathrm{BEV}$ maintenance in patients with advanced NSCLC who did not progress during or after first-line platinum-based chemotherapy. ${ }^{62}$ Median PFS was 37.1 weeks with nivolumab + BEV, with the median OS not reached (range: 33.3, 86.7+ weeks). Few cases of Grade 3 treatment-related AEs occurred, and no Grade 4 AEs occurred in the nivolumab $+\mathrm{BEV}$ arms. These preliminary data support further evaluation of the efficacy of nivolumab \pm BEV maintenance therapy in chemotherapy-treated patients. To compare the efficacy and safety of PD-1/PD-L1 blockade in combination with different treatment regimens, we have summarized that information in Table 5.

Necitumumab is a second-generation, recombinant human IgG1, EGFR mAb. Data from two randomized studies, INSPIRE and SQUIRE, indicated that necitumumab + gemcitabine and cisplatin, as a first-line therapy, could improve OS in treatment-naïve patients with advanced SqNSCLC (11.5 versus 9.9 months; $P=0.01$ ), but not in
non-SqNSCLC patients who are treated with necitumumab + pemetrexed and cisplatin. ${ }^{63,64}$ Necitumumab showed promising outcomes for patients with SqNSCLC. Recently, a Phase I study was designed to observe the safety and efficacy for necitumumab + pembrolizumab in advanced SqNSCLC and non-SqNSCLC patients. ${ }^{65}$

\section{PD- I/PD-LI blockade in combination with CTLA-4 inhibitors in NSCLC}

As mentioned, activation of T-cells requires antigen-specific stimulation via the TCR and a costimulatory signal. Among two coinhibitory signal model, CTLA-4 as the first identified coinhibitory pathway, a transmembrane glycoprotein with considerable homology to CD28, transmits an inhibitory signal to naïve T-cells and T-helper cells and enhances regulatory T-cell immunosuppressive activity. CTLA-4 inhibits T-cell activation by outcompeting CD28 in binding to CD80 and CD86. In contrast, PD-1 is primarily to inhibit activated T-cell function via its interaction with PD-L1 and PD-L2. It has been demonstrated that PD-1 ligation inhibited PI3K activity and downstream AKT activation, whereas CTLA-4 only inhibited AKT activation but did not influence PI3K activity. As CTLA-4 and PD-1 regulate immune response through a nonredundant distinct mechanism, it was possible to achieve superior antitumor effects by combined with dual blockade therapy. PD-1/PD-L1 blockades, in combination with anti-CTLA-4 mAb, as a dual checkpoint blockade has received increased attention due to the potential synergy effect. MEDI4736, an inhibitor of PD-L1, combined with tremelimumab (a selective human $\mathrm{IgG} 2 \mathrm{mAb}$ inhibitor of CTLA-4) has showed a promising response (PR and SD) with a manageable safety profile in solid tumors. ${ }^{66}$ CheckMate 067 was conducted to evaluate the efficacy and safety of nivolumab \pm ipilimumab in comparison with ipilimumab alone in patients with untreated metastatic melanoma. ${ }^{67}$ Results showed the median PFS was 11.5 months with nivolumab + ipilimumab, which was significantly high when compared with 2.9 months with ipilimumab $(P<0.001)$, and 6.9 months with nivolumab $(P<0.001)$. However, treatment-related AEs of Grade 3-4 occurred $55.0 \%$ in the nivolumab + ipilimumab group, which were also higher compared with $16.3 \%$ in the nivolumab group and $27.3 \%$ in the ipilimumab group. So far, the dosage, timing, efficacy, and safety of the combination compared to standard of care are still unknown. Recently, a dose-escalation Phase I study was designed to evaluate safety/tolerability and to identify the maximum tolerated dose of MEDI4736 in combination with tremelimumab in advanced solid tumors including NSCLC. ${ }^{68}$ A Phase III study, which is designed to evaluate the efficacy 


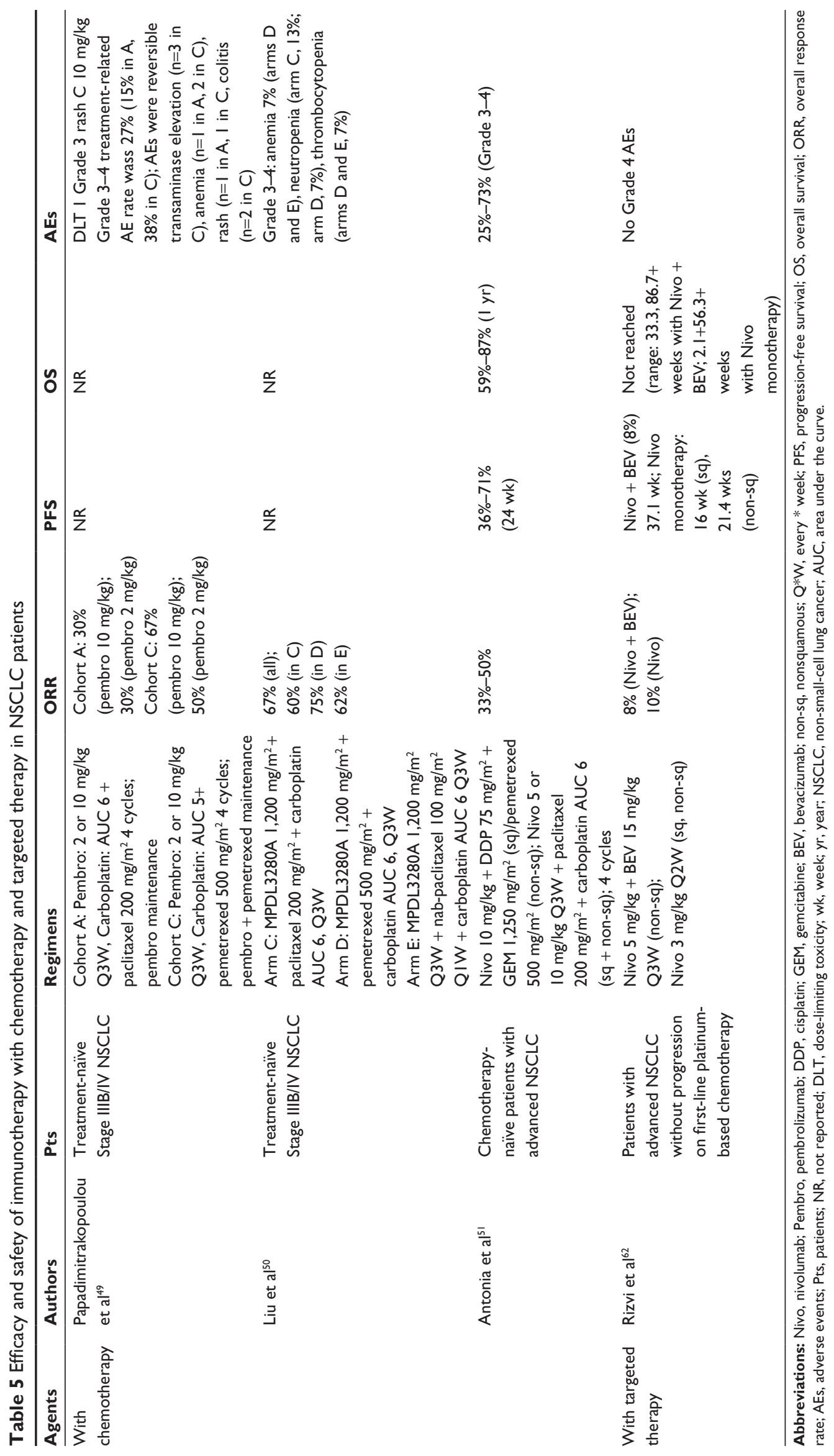


and safety of MEDI4736 as third-line monotherapy (substudy A, PD-L1 positive) + tremelimumab (substudy B, PD-L1 negative) versus standard of care (including erlotinib, gemcitabine, or vinorelbine) in Stage IIIB/IV NSCLC patients, is currently recruiting participants. ${ }^{69}$

\section{PD-I/PD-LI inhibitors in combination with radiotherapy in NSCLC}

Radiotherapy has been recognized as a local therapy. Tumor immunogenicity relapse, mediated by irradiation, has been demonstrated in several models. Preclinical studies have indicated that tumor antigen-specific $\mathrm{CD} 8^{+} \mathrm{T}$-cells increase following treatment of ablative single-dose local radiation. The complex local immune microenvironment induced by radiation enhances the host's capability of immune response to the tumor, which may result in a metastatic lesion regression. This process is the so-called abscopal effect or radiation-induced bystander effect. ${ }^{70,71}$ These complex molecular mechanisms allow combination therapy to become a possibility. ${ }^{72}$ As a result, radiotherapy combined with immunotherapy, in particular, a PD-1/PD-L1 pathway blockade, has generated a great deal of attention as a possible treatment regimen for NSCLC.

However, to date, no studies combining radiotherapy and immunotherapy have been completed for patients with NSCLC. Although it may be a new paradigm in cancer treatment, radiotherapy combined with immunotherapy still leaves a number of issues unresolved, such as the mechanism of combined therapy, the fraction model of irradiation, the time window for the combination of two treatments, and so on. ${ }^{73}$

Currently, the main research focus in radioimmunotherapy is to determine the interaction of fraction regimens and immunotherapy. CTLA-4 is another checkpoint pathway that can attenuate the early activation of naïve and memory T-cells. An abscopal effect, mediated by radiotherapy and CTLA-4 inhibitors, has been reported in a treatment-refractory lung adenocarcinoma patient. ${ }^{74}$ An immune-mediated abscopal effect has been observed with fractioned but not single-dose radiotherapy combined with anti-CTLA-4 antibody. ${ }^{75}$ Schaue et $\mathrm{al}^{76}$ also demonstrated that radiation-mediated immune response varied with the size of dose per fraction. The medium-size radiation doses of 7.5 Gy/fraction gave the best tumor control rather than singledose fractioned doses. PD-1 and PD-L1 act in a negative feedback loop to downregulate T-cell effector function and mediate apoptosis of infiltrating T-cells, leading to tumor immune evasion. ${ }^{12,77} \mathrm{We}$ believe that irradiation combined with anti-PD-L1 mAbs might also show similar results. Currently, a Phase I/II study has been designed to evaluate the safety and tolerability of pembrolizumab with SBRT in patients with NSCLC. ${ }^{78}$ The goal of Phase II of this study is to validate the benefits from combination therapy for metastatic NSCLC. This study is not yet open for participant recruitment. Because of the abscopal effect, investigators believe that local SBRT to a single lesion for recurrent NSCLC in combination with checkpoint inhibitors could enhance the antitumor immune response, which could prolong patients' PFS or OS. However, as mentioned earlier, the model of irradiation in combination with checkpoint inhibitors has not been defined. A randomized Phase II, two-arm study was designed to observe ORR to pembrolizumab after SBRT versus pembrolizumab alone in patients with advanced NSCLC. ${ }^{79}$ Another Phase I/II study was designed to evaluate the combination of pembrolizumab and SBRT in patients with metastatic NSCLC. ${ }^{80}$

\section{Conclusion}

As a result of the success using anti-PD-1/PD-L1 mAbs in clinical studies, research in the field of immunotherapy has greatly increased in recent years. Anti-PD-1 and anti-PD-L1 mAbs, used as third-, second-, and even first-line therapy, have shown promising efficacy in patients with NSCLC and has consequently impacted upon the survival and quality of life. However, immunotherapy is not perfect and has a lot of unanswered questions, such as when and how to evaluate the efficacy, what are the effective biomarkers to predict response and prognosis, and so on. Additionally, it is critical to clarify the appropriate patients who will benefit from checkpoint blockade. Furthermore, investigations to determine the mechanisms of checkpoint pathways and the efficacy of combining immunotherapy with other antitumor agents are warranted.

\section{Disclosure}

The authors report no conflicts of interest in this work.

\section{References}

1. Jemal A, Bray F, Center MM, Ferlay J, Ward E, Forman D. Global cancer statistics. CA Cancer J Clin. 2011;61(2):69-90.

2. Howington JA, Blum MG, Chang AC, Balekian AA, Murthy SC. Treatment of stage I and II non-small cell lung cancer: diagnosis and management of lung cancer, 3rd ed: American College of Chest Physicians evidence-based clinical practice guidelines. Chest. 2013 143(5 Suppl):e278S-e313S.

3. Nagata Y, Hiraoka M, Shibata T, et al. A phase II trial of stereotactic body radiation therapy for operable T1N0M0 non-small cell lung cancer: Japan clinical oncology group (JCOG0403). Int J Radiat Oncol Biol Phys. 2010;78(3):S27-S28. 
4. Spiro SG, Gould MK, Colice GL; American College of Chest P. Initial evaluation of the patient with lung cancer: symptoms, signs, laboratory tests, and paraneoplastic syndromes: ACCP evidenced-based clinical practice guidelines (2nd edition). Chest. 2007;132(3 Suppl):149S-160S.

5. Ettinger DS, Akerley W, Borghaei $\mathrm{H}$, et al. Non-small cell lung cancer. J Natl Compr Canc Netw. 2012;10(10):1236-1271.

6. Thomas A, Hassan R. Immunotherapies for non-small-cell lung cancer and mesothelioma. Lancet Oncol. 2012;13(7):e301-e310.

7. Younes RN, Pereira JR, Fares AL, Gross JL. Chemotherapy beyond first-line in stage IV metastatic non-small cell lung cancer. Rev Assoc Med Bras. 2011;57(6):686-691.

8. seer.cancer.gov [homepage on the Internet]. SEER stat fact sheets: lung and bronchus cancer [updated August 20, 2015; cited October 31, 2015]. Available from: http://seer.cancer.gov/. Accessed October 31, 2015.

9. Barlesi F, Scherpereel A, Gorbunova V, et al. Maintenance bevacizumab-pemetrexed after first-line cisplatin-pemetrexed-bevacizumab for advanced nonsquamous nonsmall-cell lung cancer: updated survival analysis of the AVAPERL (MO22089) randomized phase III trial. Ann Oncol. 2014;25(5):1044-1052.

10. National Comprehensive Cancer Network.Fort Washington.@National Comprehensive Cancer Network, Inc; 2015. Available from: http:// www.nccn.org/professionals/physician_gls/f_guidelines.asp\#nscl. Accessed October 23, 2015

11. Keir ME, Butte MJ, Freeman GJ, Sharpe AH. PD-1 and its ligands in tolerance and immunity. Апnи Rev Immunol. 2008;26:677-704.

12. Dong H, Strome SE, Salomao DR, et al. Tumor-associated B7-H1 promotes T-cell apoptosis: a potential mechanism of immune evasion. Nat Med. 2002;8(8):793-800.

13. Konishi J, Yamazaki K, Azuma M, Kinoshita I, Dosaka-Akita H, Nishimura M. B7-H1 expression on non-small cell lung cancer cells and its relationship with tumor-infiltrating lymphocytes and their PD-1 expression. Clin Cancer Res. 2004;10(15):5094-5100.

14. Okazaki T, Maeda A, Nishimura H, Kurosaki T, Honjo T. PD-1 immunoreceptor inhibits $\mathrm{B}$ cell receptor-mediated signaling by recruiting src homology 2-domain-containing tyrosine phosphatase 2 to phosphotyrosine. Proc Natl Acad Sci U S A. 2001;98(24):13866-13871.

15. Sheppard KA, Fitz LJ, Lee JM, et al. PD-1 inhibits T-cell receptor induced phosphorylation of the ZAP70/CD3zeta signalosome and downstream signaling to PKCtheta. FEBS Lett. 2004;574(1-3):37-41.

16. Lesterhuis WJ, Steer H, Lake RA. PD-L2 is predominantly expressed by Th2 cells. Mol Immunol. 2011;49(1-2):1-3.

17. Zhang Y, Huang S, Gong D, Qin Y, Shen Q. Programmed death-1 upregulation is correlated with dysfunction of tumor-infiltrating CD8+ T lymphocytes in human non-small cell lung cancer. Cell Mol Immunol. 2010;7(5):389-395.

18. Taube JM. Unleashing the immune system: PD-1 and PD-Ls in the pre-treatment tumor microenvironment and correlation with response to PD-1/PD-L1 blockade. Oncoimmunology. 2014;3(11):e963413.

19. Gettinger SN, Hellmann MD, Shepherd FA, et al. First-line monotherapy with nivolumab (NIVO; anti-programmed death-1 [PD-1]) in advanced non-small cell lung cancer (NSCLC): safety, efficacy and correlation of outcomes with PD-1 ligand (PD-L1) expression. J Clin Oncol. 2015;33(15 Suppl):8025.

20. Rizvi NA, Mazieres J, Planchard D, et al. Activity and safety of nivolumab, an anti-PD-1 immune checkpoint inhibitor, for patients with advanced, refractory squamous non-small-cell lung cancer (CheckMate 063): a phase 2, single-arm trial. Lancet Oncol. 2015;16(3):257-265.

21. Carbognin L, Pilotto S, Milella M, et al. Differential activity of nivolumab, pembrolizumab and MPDL3280A according to the tumor expression of programmed death-ligand-1 (PD-L1): sensitivity analysis of trials in melanoma, lung and genitourinary cancers. PLoS One. 2015;10(6):e0130142.

22. Garon EB, Rizvi NA, Hui R, et al. Pembrolizumab for the treatment of non-small-cell lung cancer. N Engl J Med. 2015;372(21):2018-2028.

23. Rizvi NA, Garon EB, Leighl N, et al. Optimizing PD-L1 as a biomarker of response with pembrolizumab (pembro; MK-3475) as first-line therapy for PD-L1-positive metastatic non-small cell lung cancer (NSCLC): updated data from KEYNOTE-001. J Clin Oncol. 2015;33(15 Suppl):8026.
24. Herbst RS, Soria JC, Kowanetz M, et al. Predictive correlates of response to the anti-PD-L1 antibody MPDL3280A in cancer patients. Nature. 2014;515(7528):563-567.

25. Horn L, Spigel DR, Gettinger SN, et al. Clinical activity, safety and predictive biomarkers of the engineered antibody MPDL3280A (antiPDL1) in non-small cell lung cancer (NSCLC): update from a phase Ia study. J Clin Oncol. 2015;33(15 Suppl):8029.

26. Spigel DR, Chaft JE, Gettinger SN, et al. Clinical activity and safety from a phase II study (FIR) of MPDL3280A (anti-PDL1) in PD-L1selected patients with non-small cell lung cancer (NSCLC). J Clin Oncol. 2015;33(15 Suppl):8028.

27. Merck Sharp \& Dohme Corp. Study of pembrolizumab (MK-3475) in participants with advanced non-small cell lung cancer (MK-3475025/KEYNOTE-025). Available from: https://www.clinicaltrials.gov/ ct2/show/NCT02007070. NLM identifier: NCT02007070. Accessed November 28, 2015.

28. Brahmer J, Reckamp KL, Baas P, et al. Nivolumab versus docetaxel in advanced squamous-cell non-small-cell lung cancer. $N$ Engl J Med. 2015;373(2):123-135.

29. Paz-Ares L, Horn L, Borghaei H, et al. Phase III, randomized trial (CheckMate 057) of nivolumab (NIVO) versus docetaxel (DOC) in advanced non-squamous cell (non-SQ) non-small cell lung cancer (NSCLC). J Clin Oncol. 2015;33(18 Suppl):LBA109.

30. Jiang L, Zhao Z, Jiang S, et al. Immunological markers predict the prognosis of patients with squamous non-small cell lung cancer. Immunol Res. 2015;62(3):316-324.

31. Pan ZK, Ye F, Wu X, An HX, Wu JX. Clinicopathological and prognostic significance of programmed cell death ligand1 (PD-L1) expression in patients with non-small cell lung cancer: a meta-analysis. $J$ Thorac Dis. 2015;7(3):462-470.

32. Wang A, Wang HY, Liu Y, et al. The prognostic value of PD-L1 expression for non-small cell lung cancer patients: a meta-analysis. Eur J Surg Oncol. 2015;41(4):450-456.

33. Llosa NJ, Cruise M, Tam A, et al. The vigorous immune microenvironment of microsatellite instable colon cancer is balanced by multiple counter-inhibitory checkpoints. Cancer Discov. 2015;5(1):43-51.

34. Le DT, Uram JN, Wang H, et al. PD-1 blockade in tumors with mismatchrepair deficiency. $N$ Engl J Med. 2015;372(26):2509-2520.

35. Li M, Zhang Q, Liu L, et al. Expression of the mismatch repair gene hMLH1 is enhanced in non-small cell lung cancer with EGFR mutations. PLoS One. 2013;8(10):e78500.

36. Slovakova P, Majerova L, Matakova T, Skerenova M, Kavcova E, Halasova E. Mismatch repair gene polymorphisms and association with lung cancer development. Adv Exp Med Biol. 2015;833:15-22.

37. Wolchok JD, Hoos A, O'Day S, et al. Guidelines for the evaluation of immune therapy activity in solid tumors: immune-related response criteria. Clin Cancer Res. 2009;15(23):7412-7420.

38. Nishino M, Giobbie-Hurder A, Gargano M, Suda M, Ramaiya NH, Hodi FS. Developing a common language for tumor response to immunotherapy: immune-related response criteria using unidimensional measurements. Clin Cancer Res. 2013;19(14):3936-3943.

39. Kerr KM, Tsao MS, Nicholson AG, et al. Programmed death-ligand 1 immunohistochemistry in lung cancer: in what state is this art? J Thorac Oncol. 2015;10(7):985-989.

40. Jia M, Feng W, Kang S, et al. Evaluation of the efficacy and safety of antiPD-1 and anti-PD-L1 antibody in the treatment of non-small cell lung cancer (NSCLC): a meta-analysis. J Thorac Dis. 2015;7(3):455-461.

41. Topalian SL, Hodi FS, Brahmer JR, et al. Safety, activity, and immune correlates of anti-PD-1 antibody in cancer. NEngl J Med. 2012;366(26): $2443-2454$

42. Gettinger SN, Horn L, Gandhi L, et al. Overall survival and long-term safety of nivolumab (anti-programmed death 1 antibody, BMS-936558, ONO-4538) in patients with previously treated advanced non-small-cell lung cancer. J Clin Oncol. 2015;33(18):2004-2012.

43. Bauer TM, McCleod M, Chandler JC, et al. An ongoing phase IIIb/IV safety trial of nivolumab (NIVO) in patients (pts) with advanced or metastatic non-small-cell lung cancer (NSCLC) who progressed after receiving 1 or more prior systemic regimens. J Clin Oncol. 2015;33(15 Suppl):3013. 
44. Merck Sharp \& Dohme Corp. Study of MK-3475 (pembrolizumab) versus platinum-based chemotherapy for participants with PD-L1positive advanced or metastatic non-small cell lung cancer (MK-3475042/KEYNOTE-042). Available from: https://www.clinicaltrials.gov/ ct2/show/NCT02220894. NLM identifier: NCT02220894. Accessed November 28, 2015.

45. Merck Sharp \& Dohme Corp. Study of pembrolizumab (MK-3475) compared to platinum-based chemotherapies in participants with metastatic non-small cell lung cancer (MK-3475-024/KEYNOTE-024) Available from: https://www.clinicaltrials.gov/ct2/show/NCT02142738. NLM identifier: NCT02142738. Accessed November 28, 2015.

46. Spira AI, Park K, Mazieres J, et al. Efficacy, safety and predictive biomarker results from a randomized phase II study comparing MPDL3280A vs docetaxel in 2L/3L NSCLC (POPLAR). J Clin Oncol. 2015;33(15 Suppl):8010.

47. AstraZeneca. A global study to assess the effects of MEDI4736 in patients with locally advanced or metastatic non small cell lung cancer (ATLANTIC). Available from: https://www.clinicaltrials.gov/ ct2/show/NCT02087423. NLM identifier: NCT02087423. Accessed November 28, 2015.

48. Rizvi NA, Chow LQM, Dirix LY, et al. Clinical trials of MPDL3280A (anti-PDL1) in patients (pts) with non-small cell lung cancer (NSCLC). J Clin Oncol. 2014;32(15 Suppl):TPS8123.

49. Papadimitrakopoulou V, Patnaik A, Borghaei H, et al. Pembrolizumab (pembro; MK-3475) plus platinum doublet chemotherapy (PDC) as front-line therapy for advanced non-small cell lung cancer (NSCLC): KEYNOTE-021 Cohorts A and C. J Clin Oncol. 2015;33 (15 Suppl):8031.

50. Liu SV, Powderly JD, Camidge DR, et al. Safety and efficacy of MPDL3280A (anti-PDL1) in combination with platinum-based doublet chemotherapy in patients with advanced non-small cell lung cancer (NSCLC). J Clin Oncol. 2015;33(15 Suppl):8030.

51. Antonia SJ, Brahmer JR, Gettinger SN, et al. Nivolumab (anti-PD-1; BMS-936558, ONO-4538) in combination with platinum-based doublet chemotherapy (PT-DC) in advanced non-small cell lung cancer (NSCLC). J Clin Oncol. 2014;32(15 Suppl):8113.

52. Jyoti Patel. Carboplatin/Nab-Paclitaxel and MK-3475 in NSCLC. Available from: https:/www.clinicaltrials.gov/ct2/show/NCT02382406. NLM identifier: NCT02382406. Accessed November 28, 2015.

53. Providence Health \& Services. MK-3475 and gemcitabine in non-small cell lung cancer (NSCLC). Available from: https:/www.clinicaltrials. gov/ct2/show/NCT02422381. NLM identifier: NCT02422381. Accessed November 28, 2015.

54. European Thoracic Oncology Platform. NIvolumab consolidation after standard first-line chemotherapy and radiotherapy in locally advanced stage IIIA/B NSCLC (NICOLAS). Available from: https://www. clinicaltrials.gov/show/NCT02434081. NLM identifier: NCT02434081. Accessed November 28, 2015.

55. Nasser Hanna MD. Consolidation pembrolizumab (MK-3475) following chemoradiation in patients with inoperable/unresectable stage III NSCLC. Available from: https://www.clinicaltrials.gov/ ct2/show/NCT02343952. NLM identifier: NCT02343952. Accessed November 28, 2015.

56. Chen N, Fang W, Zhan J, et al. Upregulation of PD-L1 by EGFR activation mediates the immune escape in EGFR-driven NSCLC: implication for optional immune targeted therapy for NSCLC patients with EGFR mutation. J Thorac Oncol. 2015;10(6):910-923.

57. Akbay EA, Koyama S, Carretero J, et al. Activation of the PD-1 pathway contributes to immune escape in EGFR-driven lung tumors. Cancer Discov. 2013;3(12):1355-1363.

58. D'Incecco A, Andreozzi M, Ludovini V, et al. PD-1 and PD-L1 expression in molecularly selected non-small-cell lung cancer patients. $\mathrm{Br}$ J Cancer. 2015;112(1):95-102.

59. Lin C, Chen X, Li M, et al. Programmed death-ligand 1 expression predicts tyrosine kinase inhibitor response and better prognosis in a cohort of patients with epidermal growth factor receptor mutation-positive lung adenocarcinoma. Clin Lung Cancer. 2015;16(5): e25-e35.
60. Tang Y, Fang W, Zhang Y, et al. The association between PD-L1 and EGFR status and the prognostic value of PD-L1 in advanced nonsmall cell lung cancer patients treated with EGFR-TKIs. Oncotarget. 2015;6(16):14209-14219.

61. Creelan BC, Chow LQ, Kim D-W, et al. Safety and tolerability results from a phase I study of MEDI4736, a human IgG1 anti-programmed cell death-ligand-1 (PD-L1) antibody, combined with gefitinib in patients (pts) with non-small-cell lung cancer (NSCLC). J Clin Oncol. 2015; 33(15 Suppl):3047.

62. Rizvi NA, Antonia SJ, Shepherd FA, et al. Nivolumab (Anti-PD-1; BMS-936558, ONO-4538) maintenance as monotherapy or in combination with bevacizumab (BEV) for non-small cell lung cancer (NSCLC) previously treated with chemotherapy. Int J Radiat Oncol Biol Phys. 2014;90(5):S32.

63. Thatcher N, Hirsch FR, Luft AV, et al. Necitumumab plus gemcitabine and cisplatin versus gemcitabine and cisplatin alone as first-line therapy in patients with stage IV squamous non-small-cell lung cancer (SQUIRE): an open-label, randomised, controlled phase 3 trial. Lancet Oncol. 2015;16(7):763-774.

64. Paz-Ares L, Mezger J, Ciuleanu TE, et al. Necitumumab plus pemetrexed and cisplatin as first-line therapy in patients with stage IV non-squamous non-small-cell lung cancer (INSPIRE): an open-label, randomised, controlled phase 3 study. Lancet Oncol. 2015;16(3):328-337.

65. Eli Lilly and Company. A study of the combination of necitumumab (LY3012211) and pembrolizumab (MK3475) in participants with NSCLC. Available from: https:/www.clinicaltrials.gov/ct2/ show/NCT02451930. NLM identifier: NCT02451930. Accessed November 28, 2015.

66. Callahan MK, Ott PA, Odunsi K, et al. A phase 1 study to evaluate the safety and tolerability of MEDI4736, an anti-PD-L1 antibody, in combination with tremelimumab in patients with advanced solid tumors. J Clin Oncol. 2014;32(15 Suppl):TPS3120.

67. Larkin J, Chiarion-Sileni V, Gonzalez R, et al. Combined nivolumab and ipilimumab or monotherapy in untreated melanoma. NEngl J Med. 2015;373(1):23-34

68. Ott PA, Callahan MK, Odunsi K, et al. A phase I study to evaluate the safety and tolerability of MEDI4736, an anti-programmed cell deathligand-1 (PD-L1) antibody, in combination with tremelimumab in patients with advanced solid tumors. J Clin Oncol. 2015;33(15 Suppl): TPS3099.

69. AstraZeneca. A global study to assess the effects of MEDI4736, given as monotherapy or in combination with tremelimumab determined by PD-L1 expression versus standard of care in patients with locally advanced or metastatic non small cell lung cancer (ARCTIC). Available from: https://www.clinicaltrials.gov/ct2/show/NCT02352948. NLM identifier: NCT02352948. Accessed November 28, 2015.

70. Finkelstein SE, Timmerman R, McBride WH, et al. The confluence of stereotactic ablative radiotherapy and tumor immunology. Clin Dev Immunol. 2011;2011:439752.

71. Hatzi VI, Laskaratou DA, Mavragani IV, et al. Non-targeted radiation effects in vivo: a critical glance of the future in radiobiology. Cancer Lett. 2015;356(1):34-42.

72. Melero I, Grimaldi AM, Perez-Gracia JL, Ascierto PA. Clinical development of immunostimulatory monoclonal antibodies and opportunities for combination. Clin Cancer Res. 2013;19(5):997-1008.

73. Teng F, Kong L, Meng X, Yang J, Yu J. Radiotherapy combined with immune checkpoint blockade immunotherapy: achievements and challenges. Cancer Lett. 2015;365(1):23-29.

74. Golden EB, Demaria S, Schiff PB, Chachoua A, Formenti SC. An abscopal response to radiation and ipilimumab in a patient with metastatic non-small cell lung cancer. Cancer Immunol Res. 2013;1(6):365-372.

75. Dewan MZ, Galloway AE, Kawashima N, et al. Fractionated but not single-dose radiotherapy induces an immune-mediated abscopal effect when combined with anti-CTLA-4 antibody. Clin Cancer Res. 2009; 15(17):5379-5388.

76. Schaue D, Ratikan JA, Iwamoto KS, McBride WH. Maximizing tumor immunity with fractionated radiation. Int J Radiat Oncol Biol Phys. 2012;83(4):1306-1310. 
77. Lee SJ, Jang BC, Lee SW, et al. Interferon regulatory factor-1 is prerequisite to the constitutive expression and IFN-gamma-induced upregulation of B7-H1 (CD274). FEBS Lett. 2006;580(3):755-762.

78. M.D. Anderson Cancer Center. MK-3475 and hypofractionated stereotactic radiation therapy in patients with non-small cell lung cancer (NSCLC). Available from: https://www.clinicaltrials.gov/ ct2/show/NCT02444741. NLM identifier: NCT02444741. Accessed November 28, 2015.
79. The Netherlands Cancer Institute. Pembrolizumab after SBRT versus pembrolizumab alone in advanced NSCLC (PEMBRO-RT). Available from: https://www.clinicaltrials.gov/ct2/show/NCT02492568. NLM identifier: NCT02492568. Accessed November 28, 2015.

80. Yale University. Evaluating the combination of MK-3475 and sterotactic body radiotherapy in patients with metastatic melanoma or NSCLC. Available from: https://www.clinicaltrials.gov/ct2/show/NCT02407171. NLM identifier: NCT02407171. Accessed November 28, 2015.

\section{Publish your work in this journal}

OncoTargets and Therapy is an international, peer-reviewed, open access journal focusing on the pathological basis of all cancers, potential targets for therapy and treatment protocols employed to improve the management of cancer patients. The journal also focuses on the impact of management programs and new therapeutic agents and protocols on

\section{Dovepress}

patient perspectives such as quality of life, adherence and satisfaction. The manuscript management system is completely online and includes a very quick and fair peer-review system, which is all easy to use. Visit http://www.dovepress.com/testimonials.php to read real quotes from published authors.

Submit your manuscript here: http://www.dovepress.com/oncotargets-and-therapy-journal 